\title{
Utilização de planejamento experimental no estudo da pasteurização do suco de acerola
}

Use of experimental design to study the pasteurization of acerola juice

\author{
A. P. O. Araújo ${ }^{1}$; E. C. C. Santos ${ }^{1}$; F. S. Damasceno ${ }^{2}$; T. M. Deboni ${ }^{3}$; M. S. \\ Cuevas $^{3} ;$ R. V. Mota ${ }^{4 *}$ \\ ${ }^{1}$ Departamento de Tecnologia de Alimentos, Universidade do Estado do Pará, 68550-000, Redenção-Pará, Brazil \\ ${ }^{2}$ Programa de Pósgraduação em Ciência e Tecnologia de Alimentos/Intitúto de Tecnologia, Universidade Federal do \\ Pará, 66075-900, Belém-Pará, Brazil \\ ${ }^{3}$ Departamento de Engenharia de Alimentos, Universidade de Campinas, 13635-900, Campinas-São Paulo, Brazil \\ ${ }^{4}$ Departamento de Tecnologia de Alimentos, Universidade do Estado do Pará, 68860-000, Salvaterra-Pará, Brazil
}

*vittimota@hotmail.com

(Recebido em 09 de abril de 2016; aceito em 03 de maio de 2016)

\begin{abstract}
Este trabalho objetivou estudar a pasteurização do suco de acerola (Malpighia emarginata) através de um planejamento experimental completo $2^{2}$, variando-se tempo e temperatura do tratamento térmico obtendo como resposta parâmetros físico-químicos intrínsecos ao suco de acerola. Para a elaboração do suco foram utilizadas acerolas sãs e maduras (50\%), além de água potável (40\%) e açúcar (10 \%). Na sequência, o suco foi transferido para garrafas de vidro de $500 \mathrm{~mL}$ e pasteurizados de acordo com as condições descritas no planejamento experimental completo $2^{2}$. Como resposta ao planejamento obteve-se os parâmetros físicoquímico tais como vitamina $\mathrm{C}, \mathrm{pH}$, acidez titulável, sólidos solúveis totais, açúcares redutores em glicose e açúcares não-redutores em sacarose. Através destas análises físico-químicas observou-se que o suco de acerola elaborado no presente estudo se encontra dentro dos padrões exigidos pela legislação vigente. A análise estatística demonstrou que houve uma influência significativa $(\mathrm{p} \leq 0,05)$ das variáveis de controle (tempo e temperatura) sobre a resposta vitamina C $(849,4$ a $1186,0 \mathrm{mg} / 100 \mathrm{~g})$, sendo que para as demais respostas as variações nas condições do processo de pasteurização não apresentou diferença significativa sobre as respostas, sendo os mesmos incorporados ao resíduo para os cálculos da análise de variância. Através da análise estatística, observou-se os resultados para a variável dependente vitamina $\mathrm{C}$ mostraram que as respostas experimentais foram significativas $(p \leq 0,05)$ e o teste $\mathrm{F}$ aplicado mostrou que o modelo pode ser considerado preditivo, apresentando boa reprodutibilidade e repetibilidade.

Palavras-chave: suco de acerola, planejamento experimental, físico-química
\end{abstract}

This study investigated the pasteurization of acerola (Malpighia emarginata) juice through a complete experimental design $2^{2}$, by varying the time and temperature of heat treatment obtained in response physicochemical parameters intrinsic to the acerola juice. For the preparation of juice were used fresh and mature acerola fruits $(50 \%)$, drinking water $(40 \%)$ and sugar $(10 \%)$. Subsequently, the juice was transferred to $500 \mathrm{~mL}$ glass bottles and pasteurized according to the conditions described in the experimental design $2^{2}$. In response to planning the physicochemical parameters were obtained such as vitamin $\mathrm{C}, \mathrm{pH}$, titratable acidity, total soluble solids, reducing sugars into glucose and non-reducing sugars as sucrose. Through these physical-chemical analysis it was observed that the acerola juice prepared in this study is within the standards required by law. Statistical analysis showed that there was a significant influence $(\mathrm{p} \leq 0.05)$ of the control variables (time and temperature) on the response vitamin C (849.4 to $1186.0 \mathrm{mg} / 100 \mathrm{~g}$ ), and for other answers variations in the pasteurization conditions showed no significant difference in responses, and they are incorporated to the residue for calculation of the analysis of variance. The statistical analysis showed that the experimental responses for the dependent variable vitamin $\mathrm{C}$ were significant $(\mathrm{p} \leq 0.05)$ and applying $\mathrm{F}$ test the predictive model can be considered, with good reproducibility and repeatability.

Keywords: acerola juice, experimental planning, physical-chemical 


\section{INTRODUÇÃO}

Em todo o mundo se observa um aumento expressivo no consumo de frutas tropicais por suas propriedades e funcionalidades [1]. No Brasil, a cultura da acerola (Malpighia emarginata) oferece grandes possibilidades de sucesso, devido às ações climáticas favoráveis, principalmente na parte tropical do território nacional e pelo aspecto nutricional para a saúde pública, particularmente das populações economicamente mais carentes [2].

A acerola possui elevada concentração de vitamina $\mathrm{C}$, bem como compostos fenólicos pertencentes à classe dos flavonóides que apresentam atividades antioxidantes, carotenoides, tiamina, riboflavinas, niacina, além de ferro e cálcio que podem estar presentes em sua composição de forma significativa [3,4].

A acerola pode ser consumida in natura ou processada, tanto industrialmente como artesanalmente. Dentre os produtos processados, as formas mais comuns são: o suco pronto, a polpa congelada e o suco concentrado engarrafado [5]. O abastecimento de acerola no mercado pode ser considerado como difícil pela fragilidade dos frutos, que são bastante perecíveis. Desta forma, o processamento dos frutos é imprescindível para a manutenção da cadeia produtiva dessa espécie [6].

Tanto consumidores como fabricantes de alimentos têm se tornado interessados na vitamina $\mathrm{C}$ por ser uma das mais sensíveis (incluindo as vitaminas lipossolúveis e hidrossolúveis) ao processamento e as condições de armazenamento. As vitaminas podem ser degradadas quimicamente durante o processamento e a estocagem e sua degradação está relacionada a vários fatores, tais como: $\mathrm{pH}$, oxigênio, luz, temperatura e teor de umidade ou atividade de água [7].

No que diz respeito ao processamento de alimentos, o emprego de calor é o método mais comum para aumentar a vida de prateleira dos produtos, possibilitando a inativação ou inibição do crescimento de microrganismos e enzimas. Contudo, uma série de mudanças indesejáveis ocorre nos alimentos tratados pelo calor, como a alteração no flavor, na cor e na textura, além da destruição de vitaminas [8].

Diante do exposto, este trabalho objetivou estudar a pasteurização do suco de acerola através de um planejamento experimental completo $2^{2}$, variando-se tempo e temperatura do tratamento térmico obtendo como resposta parâmetros físico-químicos intrínsecos ao suco de acerola.

\section{MATERIAL E MÉTODOS}

Foram utilizadas acerolas (Malphigia glabra Linn.) frescas, sãs e maduras, coletadas manualmente, diretamente das aceroleiras e, em seguida, transportadas para o laboratório de Tecnologia de Alimentos da Universidade do Estado do Pará, campus XV. Os frutos foram coletados na área rural de um produtor da cidade de Redenção-PA. O açúcar refinado utilizado na formulação do suco foi adquirido no comercio local.

Os frutos foram selecionados, lavados e imersos em água clorada à $120 \mathrm{ppm}$ de cloro ativo durante 15 minutos, com posterior enxague em água potável para retirada do cloro residual. Em seguida, os frutos inteiros foram homogeneizados em liquidificador doméstico com água potável e açúcar refinado na proporção de 5:4:1, respectivamente, peneirado para a separação das sementes e cascas, obtendo-se assim o suco natural adoçado (SNA). Na sequência, o mesmo foi transferido para garrafas de vidro de $500 \mathrm{~mL}$ e pasteurizados em tanque aberto (pasteurização lenta), em banho-maria sendo imediatamente resfriados após decorrido o tempo de aquecimento estabelecido para cada ensaio. Este processo decorreu-se de acordo com as condições descritas no delineamento experimental (Tabela 1).

Para avaliar os efeitos das condições de operação sobre as características físico-químicas do suco de acerola, os experimentos foram realizados com base em um planejamento experimental $2^{2}$ com três pontos centrais, tendo como variáveis independentes o tempo e a temperatura, variando em 5 diferentes níveis, totalizando 11 ensaios (Tabela 1 e 2) [9]. Tais níveis foram escolhidos através de dados obtidos por Mattietto e Matta [10], Mercali et al. [11] e Jaeschke; Marczak; Mercali [12]. 
Tabela 1: Parâmetros utilizados para a pasteurização do suco de acerola

\begin{tabular}{lccccc}
\hline \multirow{2}{*}{ Parâmetros experimentais } & \multicolumn{5}{c}{ Níveis } \\
\cline { 2 - 6 } & $\mathbf{- 1 , 4 1}$ & $\mathbf{- 1}$ & $\mathbf{0}$ & $\mathbf{1}$ & $\mathbf{1 , 4 1}$ \\
\hline Tempo $(\min )\left(\mathbf{X}_{\mathbf{1}}\right)$ & 13 & 15 & 20 & 25 & 27 \\
Temperatura $\left({ }^{\circ} \mathbf{C}\right)\left(\mathbf{X}_{\mathbf{2}}\right)$ & 70 & 74 & 84 & 94 & 98 \\
\hline
\end{tabular}

Tabela 2: Matriz do planejamento experimental

\begin{tabular}{ccccc}
\hline \multirow{2}{*}{ Ensaios } & \multicolumn{2}{c}{ Variáveis codificadas } & \multicolumn{2}{c}{ Variáveis reais } \\
\cline { 2 - 5 } & $\left(\mathbf{X}_{\mathbf{1}}\right)$ & $\left(\mathbf{X}_{\mathbf{2}}\right)$ & $\left(\mathbf{X}_{\mathbf{1}}\right)(\mathbf{m i n})$ & $\left(\mathbf{X}_{\mathbf{2}}\right)\left({ }^{\circ} \mathbf{C}\right)$ \\
\hline 1 & -1 & -1 & 15 & 74 \\
2 & 1 & 1 & 25 & 74 \\
3 & -1 & 1 & 15 & 94 \\
4 & 1 & 0 & 25 & 94 \\
5 & $-1,41$ & 0 & 13 & 84 \\
6 & 1,41 & $-1,41$ & 27 & 84 \\
7 & 0 & 1,41 & 20 & 70 \\
8 & 0 & 0 & 20 & 98 \\
9 & 0 & 0 & 20 & 84 \\
10 & 0 & 0 & 20 & 84 \\
11 & 0 & & 20 & 84 \\
\hline
\end{tabular}

Como resposta a este planejamento experimental, as variáveis dependentes (respostas) foram os teores de vitamina $\mathrm{C}$ [13], $\mathrm{pH}$ medido por aparelho phmetro de bancada, acidez titulável (AT) (porcentagem de ácido cítrico) por titulação com solução de $\mathrm{NaOH} 0,1 \mathrm{~N}$, sólidos solúveis totais (SST) medido por leitura em refratômetro com escala de 0 a $35^{\circ}$ Brix, açúcares redutores em glicose (AR) e açúcares não-redutores em sacarose (ANR), de acordo com as metodologias descritas por Instituto Adolfo Lutz [14]. Tais determinações foram realizadas, em triplicata, sendo apresentado os valores médios.

A análise estatística do estudo de pasteurização do suco de acerola foi realizada através da análise de variância (ANOVA), significância da regressão e falta de ajuste, para isso os efeitos não significativos foram incorporados aos resíduos. Os modelos foram avaliados levando em consideração os valores de $\mathrm{F}$ e coeficiente de determinação. Os dados obtidos experimentalmente foram tratados através do software Statistica ${ }^{\circledR}$.

\section{RESULTADOS E DISCUSSÃO}

A Tabela 3 apresenta as respostas do planejamento experimental com os teores de vitamina $\mathrm{C}$, pH, acidez titulável, açúcares redutores em glicose, açúcares não-redutores em sacarose e sólidos solúveis no suco de acerola pasteurizado. 
Tabela 3: Resultados da caracterização físico-química do suco de acerola pasteurizado de acordo com o planejamento experimental

\begin{tabular}{ccccccccc}
\hline Ensaio & $\begin{array}{c}\mathbf{X}_{\mathbf{1}} \\
(\mathbf{m i n})\end{array}$ & $\begin{array}{c}\mathbf{X}_{\mathbf{2}} \\
\left({ }^{\circ} \mathbf{C}\right)\end{array}$ & $\begin{array}{c}\text { Vitamina C } \\
(\mathbf{m g} / \mathbf{1 0 0} \mathbf{g})\end{array}$ & $\mathbf{p H}$ & $\mathbf{A T}(\boldsymbol{\%})$ & $\mathbf{A R}(\boldsymbol{\%})$ & ANR $(\%)$ & SST $\left({ }^{\circ} \mathbf{B r i x}\right)$ \\
\hline 1 & 15 & 74 & $1174,5 \pm 0,6$ & $3,26 \pm 0,04$ & $0,92 \pm 0,020$ & $5,26, \pm 0,005$ & $2,74 \pm 0,011$ & $13,00 \pm 0,005$ \\
2 & 25 & 74 & $1148,6 \pm 0,4$ & $3,28 \pm 0,01$ & $0,91 \pm 0,017$ & $5,21 \pm 0,020$ & $2,86 \pm 0,020$ & $13,03 \pm 0,020$ \\
3 & 15 & 94 & $886,7 \pm 0,5$ & $3,42 \pm 0,01$ & $0,74 \pm 0,017$ & $4,68 \pm 0,010$ & $3,36 \pm 0,011$ & $13,11 \pm 0,035$ \\
4 & 25 & 94 & $861,7 \pm 0,3$ & $3,50 \pm 0,01$ & $0,66 \pm 0,020$ & $4,32 \pm 0,011$ & $3,5 \pm 0,005$ & $13,14 \pm 0,030$ \\
5 & 13 & 84 & $1101,4 \pm 0,5$ & $3,32 \pm 0,01$ & $0,83 \pm 0,030$ & $5,15 \pm 0,020$ & $2,94 \pm 0,011$ & $13,06 \pm 0,010$ \\
6 & 27 & 84 & $931,6 \pm 0,3$ & $3,39 \pm 0,01$ & $0,77 \pm 0,017$ & $4,73 \pm 0,050$ & $3,24 \pm 0,010$ & $13,05 \pm 0,010$ \\
7 & 20 & 70 & $1186,0 \pm 1,1$ & $3,25 \pm 0,01$ & $0,90 \pm 0,026$ & $5,30 \pm 0,050$ & $2,64 \pm 0,011$ & $13,00 \pm 0,005$ \\
8 & 20 & 98 & $849,4 \pm 0,6$ & $3,53 \pm 0,01$ & $0,63 \pm 0,020$ & $4,19 \pm 0,020$ & $3,75 \pm 0,015$ & $13,15 \pm 0,030$ \\
9 & 20 & 84 & $1016,2 \pm 0,7$ & $3,33 \pm 0,01$ & $0,85 \pm 0,010$ & $4,88 \pm 0,005$ & $3,10 \pm 0,200$ & $13,07 \pm 0,011$ \\
10 & 20 & 84 & $1016,4 \pm 0,6$ & $3,32 \pm 0,01$ & $0,84 \pm 0,020$ & $4,87 \pm 0,015$ & $3,10 \pm 0,110$ & $13,07 \pm 0,020$ \\
11 & 20 & 84 & $1016,5 \pm 0,3$ & $3,33 \pm 0,01$ & $0,83 \pm 0,020$ & $4,87 \pm 0,015$ & $3,10 \pm 0,200$ & $13,07 \pm 0,011$ \\
\hline
\end{tabular}

O teor de ácido ascórbico encontrado no suco de acerola pasteurizado variou de 849,4 mg/100 g (ensaio 8) a 1186,0 mg/100 g (ensaio 7), podendo observar uma variação em torno de $28 \%$ de vitamina $\mathrm{C}$ entre as condições extremas de temperatura. Segundo dados da literatura este teor apresenta-se de forma bastante variada no suco de acerola, sendo descrito teores de 593,8 mg/100 $\mathrm{g}$ [15] a 2018,67 mg/100 g [16], ainda de acordo com este mesmo autor a variação nos teores de vitamina $\mathrm{C}$ pode ser decorrente de muitos fatores, tais como, variedade, grau de maturação, local de plantio, época de plantio e colheita, entre outros. Caetano; Daiuto; Vieites [17] encontraram teores de $1054 \mathrm{mg} / 100 \mathrm{~g}$ de ácido ascórbico tanto na polpa como no suco de acerola. Já Mercali et al. [18] descrevem teores de ácido ascórbico que variam de 1000 a 4000 mg/100 g de polpa de acerola fresca.

A variação do conteúdo de vitamina $\mathrm{C}$ nas amostras que passaram por algum processamento pode ser devida à oxidação química da vitamina $\mathrm{C}$ e/ou degradação térmica durante as etapas de branqueamento, cozimento, pasteurização e/ou esterilização dos sucos [19, 20]. Mesmo com a diminuição do teor de vitamina $\mathrm{C}$ durante o processamento, o alto teor desta vitamina na acerola possibilita sua industrialização sem grandes variações nutricionais.

Nos sucos que passaram pelo tratamento térmico, observou-se uma diminuição da acidez e aumento do $\mathrm{pH}$ a medida que a temperatura de pasteurização aumentou. A tendência de redução da acidez também foi evidenciada por Freitas [21] e Maia et al. [15], quando estudaram o efeito do processamento sobre componentes do suco de acerola. Tais alterações podem ser consequência da degradação dos ácidos (ácido cítrico, ácido málico, ácido ascórbico) presentes durante o tratamento térmico.

As análises de açúcares redutores em glicose e açúcares não-redutores em sacarose no suco de acerola pasteurizado do presente estudo encontram-se próximos aos valores descritos na caracterização físico-química do suco de acerola a $13{ }^{\circ}$ Brix, realizado por Silva et al. [16], que foram de 6,07 \% para açúcares redutores e 2,35 \% para não-redutores. O resultado encontrado na determinação de sólidos solúveis do suco de acerola natural está de acordo com os padrões estabelecidos pela legislação para suco tropical de acerola adoçado [22], que estabelece valores mínimos de $10{ }^{\circ}$ Brix. 
A partir dos resultados encontrados para o planejamento experimental, foi realizada a análise de variância (ANOVA) através do software Statistica ${ }^{\circledR}$, para avaliar os efeitos das variáveis de controle (tempo e temperatura) sobre as respostas (vitamina C, pH, AT, AR, ANR, SST variáveis dependentes). A análise estatística demonstrou que houve uma influência significativa $(\mathrm{p} \leq 0,05)$ das variáveis de controle (tempo e temperatura) sobre a resposta vitamina $\mathrm{C}$, sendo que para as demais respostas as variações nas condições do processo de pasteurização não apresentaram diferença significativa sobre as respostas, sendo os mesmos posteriormente incorporados ao resíduo.

Na Tabela 4 são apresentados os efeitos das variáveis controle e sua interação sobre a resposta vitamina C.

Tabela 4: Efeitos e interação calculadas para a variável vitamina $C$

\begin{tabular}{lcccc}
\hline & Efeito & Erro Puro & $\mathbf{t}(\mathbf{5})$ & P \\
\hline Média & 1016,357 & 19,473 & 52,192 & 0,0000 \\
Tempo (L) & $-72,746$ & 23,850 & $-3,050$ & 0,028 \\
Tempo (Q) & 0,546 & 28,388 & 0,019 & 0,985 \\
Temperatura (L) & $-262,653$ & 23,850 & $-11,013$ & 0,0001 \\
Temperatura (Q) & 1,726 & 28,388 & 0,061 & 0,954 \\
Tempo x temperatura & 0,460 & 33,729 & 0,014 & 0,990 \\
\hline
\end{tabular}

Os coeficientes de regressão representam a metade do efeito e foram obtidos a $95 \%$ de confiança. A análise estatística dos dados experimentais demonstrou efeitos significativos para os termos tempo e temperatura linear. Os termos não significativos foram incorporados ao resíduo para o cálculo da ANOVA, apresentada na Tabela 5.

Tabela 5: Análise de variância para a variável vitamina $C$

\begin{tabular}{lcccccc}
\hline \multicolumn{1}{c}{ Fonte de variação } & S.Q & G.L & Q.M & $\mathbf{F}_{\text {cal. }}$ & $\mathbf{F}_{\text {tab. }}$ & $\mathbf{R}^{\mathbf{2}} \mathbf{( \% )}$ \\
\hline Regressão & 148556,6 & 2 & 74278,3 & 104,4 & 4,92 & 96,3 \\
Resíduo & 5692,6 & 8 & 711,6 & & & \\
Total & 154249,2 & 10 & & & & \\
\hline
\end{tabular}

S.Q - Soma de quadrados; G.L - Graus de liberdade; Q.M - Quadrado médio; $F_{\text {cal }}$ - distribuição de F

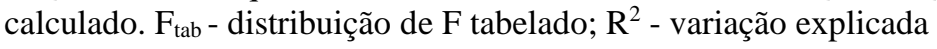

$O F_{\text {cal. }}$ para regressão $(104,4)$ foi bem superior ao $F_{\text {tab. }}(4,92)$ e, portanto, significativo. De acordo com Box et al. [23], o modelo é estatisticamente significativo quando F calculado é cerca de cinco vezes superior que o $\mathrm{F}$ tabelado, para a regressão. Com $\mathrm{R}^{2}$ de 0,963 conclui-se que o modelo se ajusta bem aos dados experimentais. Assim, obteve-se o modelo reparametrizado (Equação 1) e sua respectiva superfície de resposta e curvas de contorno (Figura 1), que descrevem de forma significativa o processo em questão.

Vitamina $C(m g / 100 m L)=1017,2-36,4\left(X_{1}\right)-131,4\left(X_{2}\right)$

(Eq. 1)

Onde:

$$
\begin{aligned}
& X_{1}=\text { tempo }(\min ) \\
& X_{2}=\text { temperatura }\left({ }^{\circ} \mathrm{C}\right)
\end{aligned}
$$



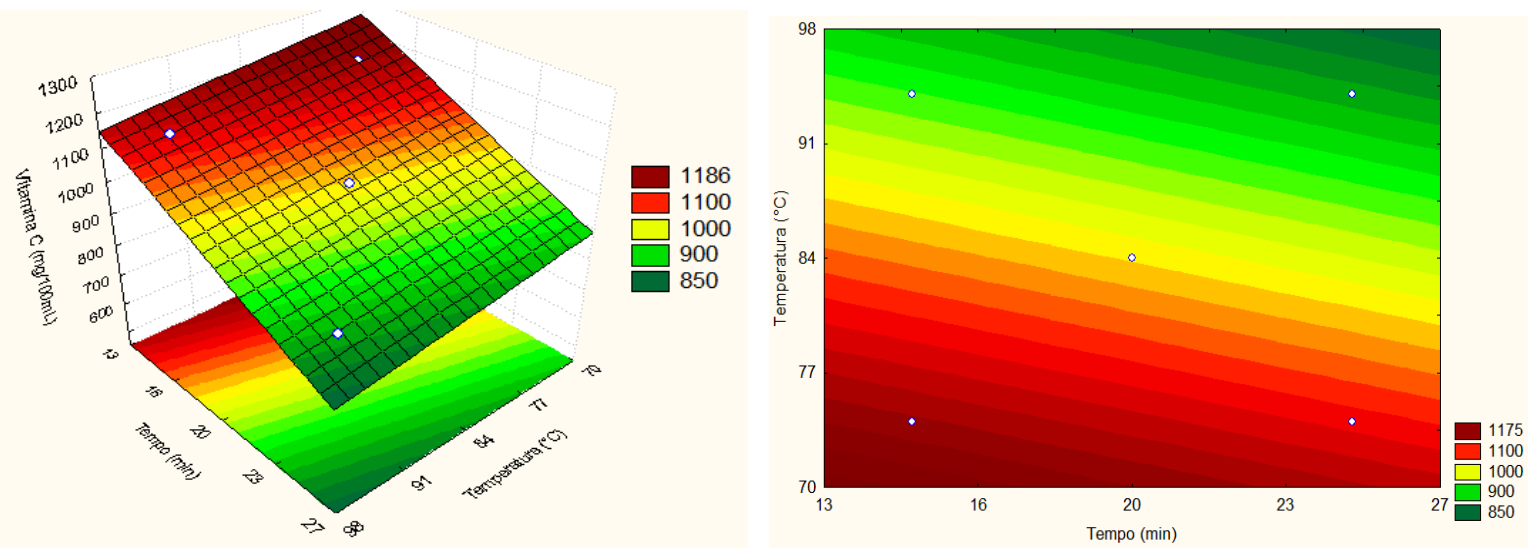

Figura 1: Superfície de resposta e curvas de contorno para a variável vitamina $C$

Observa-se que quando são aplicadas temperaturas mais baixas (zona em vermelho), mantendo-se a pasteurização em qualquer faixa de tempo estudado (13 a 27 minutos), tem-se uma maior manutenção do teor de vitamina C.

Na Tabela 6 são apresentados os valores experimentais e os preditos pelo modelo para o teor de vitamina C, os erros de ajuste (E.A.) ou erro absoluto e os erros relativos (E.R.) para cada ensaio do planejamento experimental.

Tabela 6: Valores de vitamina C experimentais, preditos pelo modelo e desvios para o planejamento

\begin{tabular}{ccccccc}
\hline Ensaios & $\mathbf{X}_{\mathbf{1}}(\mathbf{m i n})$ & $\mathbf{X}_{\mathbf{2}}\left({ }^{\circ} \mathbf{C}\right)$ & $\begin{array}{c}\text { Vit. C exp. } \\
(\boldsymbol{\%})\end{array}$ & $\begin{array}{c}\text { Vit. C pred. } \\
(\boldsymbol{\%})\end{array}$ & $\begin{array}{c}\text { E.A. } \\
(\boldsymbol{\%})\end{array}$ & E.R. $(\boldsymbol{\%})$ \\
\hline 1 & 15 & 74 & 1174,5 & 1185 & $-10,50$ & $-0,89$ \\
2 & 25 & 74 & 1148,6 & 1112,2 & 36,40 & 3,17 \\
3 & 15 & 94 & 886,7 & 922,2 & $-35,50$ & $-4,00$ \\
4 & 25 & 94 & 861,7 & 849,4 & 12,30 & 1,43 \\
5 & 13 & 84 & 1101,4 & 1068,52 & 32,88 & 2,98 \\
6 & 27 & 84 & 931,6 & 965,88 & $-34,28$ & $-3,68$ \\
7 & 20 & 70 & 1186 & 1202,474 & $-16,47$ & $-1,39$ \\
8 & 20 & 98 & 849,4 & 831,926 & 17,47 & 2,06 \\
9 & 20 & 84 & 1016,2 & 1017,2 & $-1,00$ & $-0,10$ \\
10 & 7,90 & 6,0 & 1016,4 & 1017,2 & $-0,80$ & $-0,08$ \\
11 & 7,72 & 6,0 & 1016,5 & 1017,2 & $-0,70$ & $-0,07$ \\
\hline
\end{tabular}

exp. - experimental; pred. - predito; E.A. - Erro de ajuste; E.R. - Erro relativo

Nota-se que os erros relativos foram baixos em todos os ensaios (inferior a $4 \%$ ), mostrando que o modelo que descreve a perda de vitamina $\mathrm{C}$ no suco de acerola submetido a pasteurização do presente estudo é preditivo. Através dos pontos centrais é possivel verificar que os dados obtidos neste planejamento experimental apresentam boa repetibilidade, com a diferença entre os pontos centrias (ensaios 9,10 e 11) sendo quase nula.

\section{CONCLUSÃO}

Os valores dos parâmetros físico-químicos analisados para o suco de acerola pasteurizado estão de acordo com o que preconiza a legislação vigente para este tipo de produto, bem como em consonância com dados disponíveis na literatura.

Com exceção da vitamina $\mathrm{C}$, para todas as demais variáveis não foi observado variações significativas em suas respostas, não sendo possível dar seguimento ao tratamento estatístico com a realização da análise de variância, obtenção do modelo matemático e respectiva superfície de resposta. 
Os resultados para a variável dependente vitamina $\mathrm{C}$ mostraram que as respostas experimentais, após tratamento por análise de regressão múltipla, foram significativas $(p \leq 0,05)$ e o teste $\mathrm{F}$ aplicado mostrou que o modelo pode ser considerado preditivo, apresentando boa reprodutibilidade e repetibilidade.

Em função dos resultados e do gráfico de tendência gerado, considerando-se que o tempo apresentou menor impacto sobre a resposta vitamina $\mathrm{C}$, sugere-se o desenvolvimento de novos estudos, adotando-se tempos mais reduzidos, tendo como finalidade a diminuição da exposição da vitamina $\mathrm{C}$ ao calor, bem como na redução dos custos de produção.

\section{REFERÊNCIAS BIBLIOGRÁFICAS}

1. Lima RMT. Avaliação da estabilidade química, físico-química e microbiológica de polpas de acerola orgânica pasteurizada e não-pasteurizada. [dissertação]. Fortaleza (CE): Mestrado em Ciência e Tecnologia de Alimentos: Universidade Federal do Ceará; 2010. 94p.

2. Souza MJH, Guimarães MCA, Guimarães CDL, Freitas WS, Oliveira AMS. Potencial agroclimático para a cultura da acerola no estado de Minas Gerais. Revista Brasileira de Engenharia Agrícola e Ambiental: Campina Grande. Jun. 2006; 10 (2): 390-396. doi: 10.1590/S1415-43662006000200021

3. Neves MVM, Lima VLAG. Efeito do congelamento sobre a estabilidade da polpa de acerola adicionada de extrato comercial de própolis. Alimentação e Nutrição, Araraquara. 2009; 20 (1): 87-94.

4. Godoy RCB, Matos ELS, Amorim TS, Neto MAS, Ritzinger R, waszczynskyj N. Avaliação de genótipos de variedades de acerola para consumo in natura e para elaboração de doces. Boletim CEPPA: Curitiba. 2008 Jul/Dez; 26 (2): 197-204. doi.org/10.5380/cep.v26i2.13274

5. Rojas AM, Gerschenson, LN. Ascorbic acid destruction in aqueous model systems: an additional discussion. Journal of the Science of Food and Agriculture. Out. 2001; 81(15): 1433-39, doi: $10.1002 /$ jsfa.961.

6. Gomes JE, Perecin D, Martins ABG, Fontes SR. Comportamento de propriedades físicas, químicas e reológicas do suco de acerola armazenado a baixa temperatura. Revista Brasileira de Engenharia Agrícola e Ambiental: Campina Grande. Mai/Ago. 2001; 05(2): 293-300. doi: 10.1590/S141543662001000200020

7. Elez-Martínez P, Martín BO. Effects of high intensity pulsed electric field processing conditions on vitamin $\mathrm{C}$ and antioxidant capacity of orange juice and gazpacho, a cold vegetable soup. Food Chemistry. 2007; 102 (1): 201-209, doi:10.1016/j.foodchem.2006.04.048.

8. Rodrigues MI, Iemma AF. Planejamento de Experimentos e Otimização de Processos: uma estratégia seqüencial de experimentos. $3^{\circ}$ ed., Editora Casa do Pão, Campinas (SP), 2014

9. Mattietto RA, Matta VM. Utilização de um Delineamento Composto Central Rotacional Para Avaliação Microbiológica de Polpas de Açaí Pasteurizadas. Búzios, RJ, 2012.urgh RH. Statistics in medicine. 2nd ed. Amsterdam (Netherlands): Elsevier Academic Press; 2006. Chapter 24, Regression and correlation methods; p. 447-86, doi: 10.1016/B978-0-12-384864-2.00025-1.

10. Caetano PK. Processamento Tecnológico e Avaliação Energética de Geléia de Acerola. [dissertação]. Botucatu (SP): Mestrado da Faculdade de Ciências Agronômicas: Universidade Estadual Paulista "Julio de Mesquita Filho"; 2010. p. 101.

11. Mercali GD, Jaeschke DP,Tessaro IC, Marczak LDF. Degradation kinetics of anthocyanins in acerola pulp: Comparison between ohmic and conventional heat treatment. Food Chemistry. 2013. Jan; 136(2): 853-857. doi:10.1016/j.foodchem.2012.08.024

12. Jaeschke DP, Marczak LDF, Mercali GD. Evaluation of non-thermal effects of electricity on ascorbic acid and carotenoid degradation in acerola pulp during ohmic heating. Food Chemistry. 2016 May; 199: 128-134. doi:10.1016/j.foodchem.2015.11.117.

13. Cecchi HM. Fundamentos teóricos e práticos em análise de alimentos. $2^{\circ}$ ed, Campinas (SP). 2003 Editora Unicamp.

14. INSTITUTO ADOLFO LUTZ. Métodos físico-químicos para análise de alimentos. $4^{\mathrm{a}}$ Edição $-1^{\mathrm{a}}$ Edição Digital. 2008. São Paulo (SP).

15. Maia GA, Sousa PHM, Santos GM, Silva DS, Fernandes AG, Prado GM. Efeito do processamento sobre componentes do suco de acerola. Ciência e Tecnologia de Alimentos, Campinas. 2007. Jan./mar; 27(1): 130-134. doi: 10.1590/S0101-20612007000100023.

16. Silva FC, Guimarães DHP, Gasparetto CA. Reologia do suco de acerola: efeitos da concentração e temperatura. Ciência e Tecnologia de Alimentos, Campinas-SP. 2005 jan./mar; 25(1): 121-126. 10.1590/S0101-20612005000100020. 
17. Caetano PK, Daiuto ER, Vieites RL. Característica físico-química e sensorial de geleia elaborada com polpa e suco de acerola. Brazilian Journal of food technology, Campinas-SP. 2012 jul/set; 15(3): 191197. doi: 10.1590/S1981-6723201200500001.

18. Mercali GD, Schwartz S, Marczak LDF, Tessaro IC, Sastry S. Ascorbic acid degradation and color changes in acerola pulp during ohmic heating: Effect of electric field frequency. Journal of Food Engineering. 2014; 123(1): 1-7. doi:10.1016/j.jfoodeng.2013.09.011.

19. Polydera AC, Stoforos NG, Taoukis PS. Quality degradation kinetics of pasteurized and high pressure processed fresh Navel orange juice: Nutritional parameters and shelf life. Innovative Food Science \& Emerging Technologies. 2005; 6(1): 1-9. doi:10.1016/j.ifset.2004.10.004.

20. Vikram VB, Ramesh MN, Prapulla SG. Thermal degradation kinetics of nutrients in orange juice heated by electromagnetic and conventional methods. Journal of Food Engineering. 2005; 69(1): 31-40. doi:10.1016/j.jfoodeng.2004.07.013

21. Freitas CAS, Maia GA, Costa JMC, Figueiredo RW, Sousa PHM, Fernandes AG. Estabilidade dos carotenoides, antocianinas e vitamina $\mathrm{C}$ presente no suco tropical de acerola (Malpighia emarginata DC.) adoçado envasado pelos processos hot-fill e asséptico. Ciências e Agrotecnologia: Lavras. 2006; 30(5): 942-949. doi.org/10.1590/S1413-70542006000500018.

22. Brasil, Ministério da Agricultura, Pecuária e Abastecimento. Instrução Normativa $n^{\circ} 12$ de 4 de setembro de 2003. Regulamento Técnico para fixação dos padrões de identidade e qualidade gerais para suco tropical. Disponível em: <http://www.aladi.org/nsfaladi/normasTecnicas.nsf>. Acesso em: set. 2015.

23. Box GEP, Hunter WG, Hunter JS. Statistics for Experimenters: Design, Innovation, and Discovery, 2nd Edition. New York: Wiley Series in Probability and Statistics, 2005. 